\title{
Directly Counting Number of Atoms in Au Nanoparticles Supported on Gamma-Alumina by Quantitative-STEM
}

\author{
Long Li, Matthew K. France, and Judith C. Yang \\ Department of Chemical and Petroleum Engineering, Department of Physics, University \\ of Pittsburgh, Pittsburgh, PA 15261
}

Supported metallic nanoparticles (NPs) are widely used as nanocatalysts, and the performance depends intimately to their supported 3-D structures. Quantitative measurements from STEM high-angle annular dark-field (HAADF) imaging can provide 3 -D information for supported metal NPs $[1,2]$. The measured absolute integral intensity displayed monotonic dependence on the NP size, for Au NPs up to 6500 atoms [3]. The scattering cross-section from individual NPs were quantitatively measured $[2,4]$, and the number of atoms, e.g. in $\mathrm{Re}_{6} \mathrm{NPs}$, was counted using a dedicated VG-STEM via comparison of the integral intensity and the theoretical electron scattering factor of an atom [4]. This quantitative STEM (QSTEM) technique is based on the experimental integral intensity from NPs, and it can be achieved in a STEM with intermediate resolution (around $0.2 \mathrm{~nm}$ ). We recently have achieved this technique on JEM-2100F based on no any modifications [5]. In QSTEM, HAADF detector efficiency is first accurately determined regarding to true trajectories of scattered electrons striking the detector. The HAADF detector image was taken under its linear response range using a fine and low current probe in an angle scan mode under free lens control. The scanning angle was calibrated with a STEM electron diffraction pattern from $\mathrm{Au}$ (100). The half detector inner and outermost angles were 100.3 and $252.1 \mathrm{mrad}$, respectively.

Commercial $\gamma-\mathrm{Al}_{2} \mathrm{O}_{3}$ powders were spread on ultra-thin $\mathrm{C}$-grids, and $\mathrm{Au}$ NPs were then evaporated on to such grids in an UHV e-beam system. The grids were heated at $275{ }^{\circ} \mathrm{C}$, and $\mathrm{Au}$ NPs were created through self-assembly on both $\gamma-\mathrm{Al}_{2} \mathrm{O}_{3}$ supports and the carbon film. As $\gamma-\mathrm{Al}_{2} \mathrm{O}_{3}$ contributed to the HAADF imaging as background, which should be subtracted in order to obtain the true integral intensity of each Au NP for QSTEM measurements. A circle of two pixel expansion around a NP was selected for the background estimate [3, 5], as illustrated in Fig. 1, based on symmetric background (c). The asymmetric (f) case from NPs seated at the edge of $\gamma-\mathrm{Al}_{2} \mathrm{O}_{3}$ supports can lead errors and should be avoided. The NP diameter was measured from the full width at half maximum of the intensity profile across the NP, and/or based on the average of the long and short diameters in case the NP is not round in shape. Fig. 2(a) is a selected HAADF image of Au NPs taken under the QSTEM imaging conditions. The beam current was measured with the GIF CCD camera. The numbers of atoms and diameters of individual $\mathrm{Au}$ NPs have been measured, e.g. labeled in Fig. 2(a). The entire measurements are presented in histograms for number of atoms in Fig. 2(b) and size of the Au NPs in Fig.2(c). Fig.2 (d) is a plot of the number of atoms vs. the diameters of Au NPs to show their 3-D shapes. The average size of Au NPs is $0.9 \pm 0.2 \mathrm{~nm}$ with average of $17 \pm 11 \mathrm{Au}-$ atoms. The large range ( \pm 11 atoms) of the average atom number for Au NPs indicates a variety of 3-D shapes of Au NPs even though they have a relatively narrow size distribution at the 2-D projection (diameter around $0.9 \mathrm{~nm}$ ), e.g. the difference in the number of atoms between spherical and hemi-spherical nanoparticle is a factor of two. 
Our QSTEM technique with single frame of HAADF images and measured probe current can directly provide structure information of diameters with corresponded numbers of atoms in NPs. Therefore the 3-D structure of the supported metal NP can be determined through modeling from these two key parameters.

\section{References}

[1] J. C. Yang et al., Chem. Soc. Rev. 41 (24) (2012) 8179.

[2] M. M. J. Treacy and S. B. Rice, Journal of Microscopy 156 (2) (1989) 211.

[3] N. P. Young et al., Phys. Rev. Lett. 101 (24) (2008) 246103.

[4] A. Singhal, J. C. Yang, and J. M. Gibson, Ultramicroscopy 67 (1-4) (1997) 191.

[5] L. Li, Z. Zhang, and J. C. Yang, Microsc.Microanal. 18 (S2) (2012) 1040.

[6] DOE-BES: DE-FG02-03ER15476, JEM-2100F at NFCF, University of Pittsburgh.

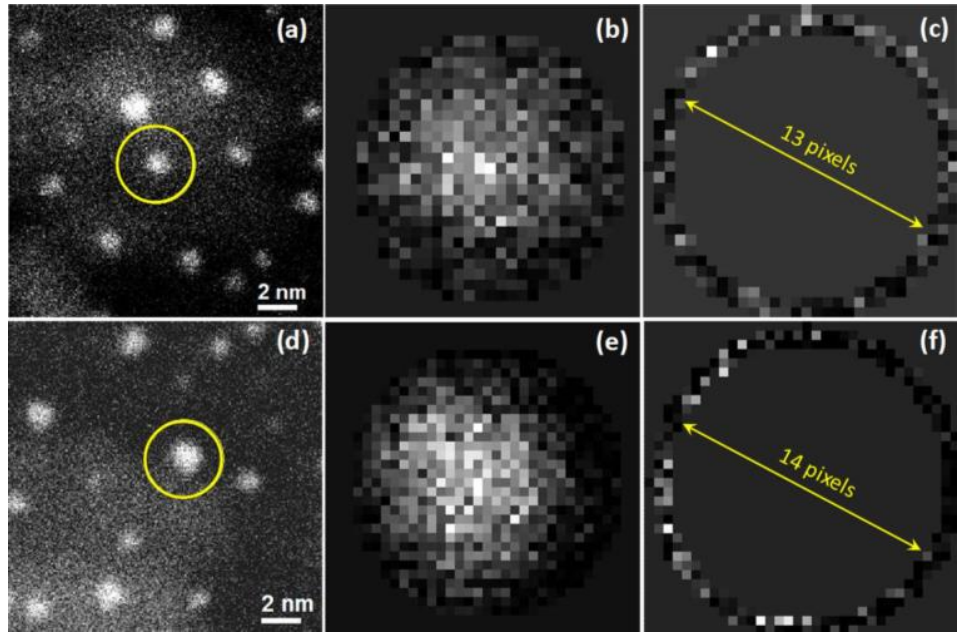

Fig. 1. Background estimate: Symmetric background (c) from a $\mathrm{Au} \mathrm{NP} / \gamma-\mathrm{Al}_{2} \mathrm{O}_{3}$ in (a). The $\mathrm{Au}$ NPs seating at edge of $\gamma-\mathrm{Al}_{2} \mathrm{O}_{3}$ in (d) should be avoided as the asymmetric background (f) can lead errors. (b) and (e) are the measured regions for Au NPs.
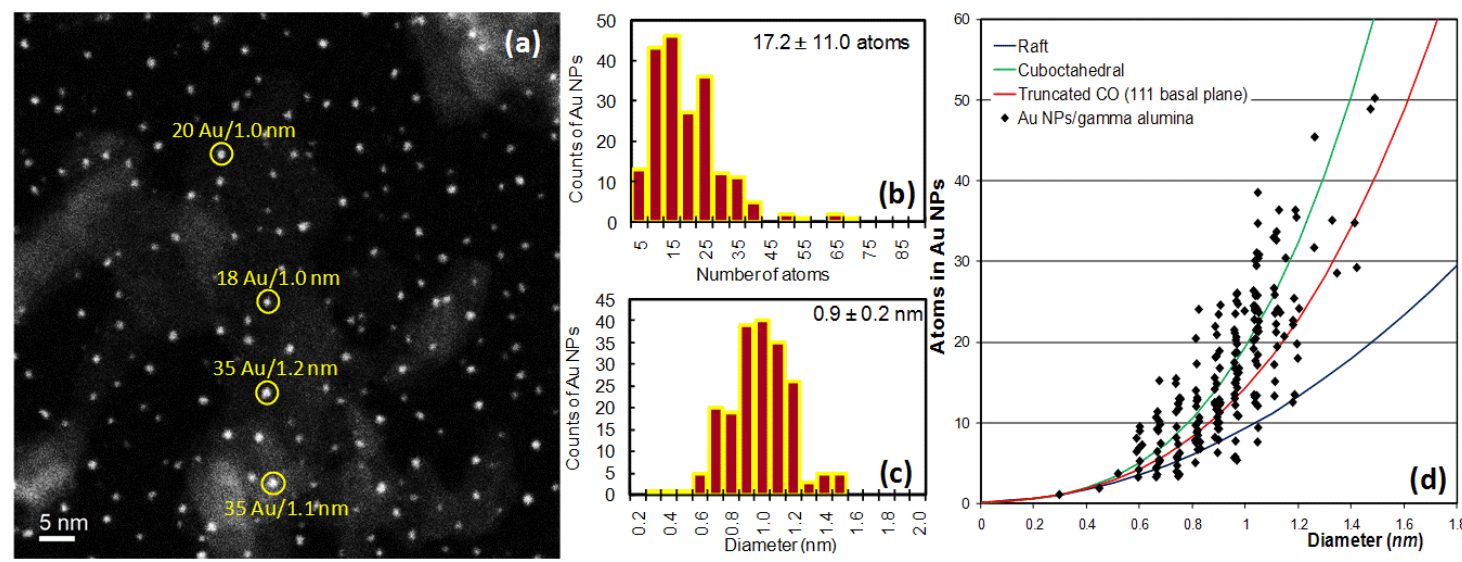

Fig. 2. (a) A representative HAADF image of $\mathrm{Au} \mathrm{NPs} / \gamma-\mathrm{Al}_{2} \mathrm{O}_{3}$, and sizes and numbers of $\mathrm{Au}$ atoms in $\mathrm{Au}$ NPs were measured via QSTEM; The histograms of atom numbers (b) and sizes (c) of Au NPs. The number of atoms vs. the diameters of Au NPs (d) revealed various 3-D structures of Au NPs self-assembled during the e-beam evaporation. 